\title{
An analytical kinetic model for chemical-vapor deposition of pureB layers from diborane
}

\author{
V. Mohammadi, ${ }^{\text {a) }}$ W. B. de Boer, and L. K. Nanver \\ Delft Institute of Microsystems and Nanoelectronics, Delft University of Technology, Feldmannweg 17, \\ 2628 CT Delft, The Netherlands
}

(Received 20 June 2012; accepted 26 October 2012; published online 3 December 2012)

\begin{abstract}
In this paper, an analytical model is established to describe the deposition kinetics and the deposition chamber characteristics that determine the deposition rates of pure boron (PureB-) layers grown by chemical-vapor deposition (CVD) from diborane $\left(\mathrm{B}_{2} \mathrm{H}_{6}\right)$ as gas source on a nonrotating silicon wafer. The model takes into consideration the diffusion mechanism of the diborane species through the stationary boundary layer over the wafer, the gas phase processes and the related surface reactions by applying the actual parabolic gas velocity and temperature gradient profiles in the reactor. These are calculated theoretically and also simulated with FLUENT software. The influence of an axial and lateral diffusion of diborane species and the validity of the model for laminar flow in experimental CVD processes are also treated. This model is based on a wide range of input parameters, such as initial diborane partial pressure, total gas flow, axial position on the wafer, deposition temperature, activation energy of PureB deposition from diborane, surface $\mathrm{H}$-coverage, and reactor dimensions. By only adjusting these reactor/process parameters, the model was successfully transferred from the ASM Epsilon One to the Epsilon 2000 reactor which has totally different reactor conditions. The model's predictive capabilities have been verified by experiments performed at $700{ }^{\circ} \mathrm{C}$ in these two different ASM CVD reactors. (C) 2012 American Institute of Physics. [http://dx.doi.org/10.1063/1.4767328]
\end{abstract}

\section{INTRODUCTION}

The chemical-vapor deposition of pure boron has in the last years been very successfully applied for fabricating extremely shallow, less than 10 -nm deep, silicon $p^{+} n$ junction diodes for a number of leading-edge device applications. ${ }^{1}$ This so-called PureB technology has provided particularly impressive performance of photodiode detectors for low penetration-depth beams for which 2-nm-thick PureB-layers are reliably implemented as the front-entrance window. ${ }^{2-5}$ Ideal low-leakage diode characteristics are achieved for deposition temperatures from $400{ }^{\circ} \mathrm{C}$ to $700{ }^{\circ} \mathrm{C}$, which together with the fact that the deposition is conformal and highly selective to $\mathrm{Si}$, also makes PureB technology an attractive candidate for creating junctions on silicon nanowires and advanced CMOS transistors including source/drain in p-type FinFETs. ${ }^{6,7}$ In the latter applications, sub-3-nm thick layers are required to avoid excess series resistance through the high-resistivity boron layer. Moreover, for the photodiode application, any thickness variations even in the angstrom range can have a large impact on the responsivity to beams that only penetrate a few nm into the Si such as VUV light and less-than $1 \mathrm{keV}$ electrons. Therefore, a very good control of the layer thickness is crucial.

The PureB deposition is susceptible to loading effects and strongly depends to the deposition and reactor conditions. ${ }^{8}$ In order to achieve better control of the deposition and an understanding of the associated kinetics, the deposition mechanism and growth characteristics should be

\footnotetext{
${ }^{a)}$ E-mail: v.mohammadi@tudelft.nl. Phone: +31 (0)15 27 86294, Fax: +31 (0)152787369.
}

described. A CVD process proceeds through several steps essentially composed of the reactant transfer in the gas phase and chemical reactions at the substrate surface. ${ }^{9}$ Therefore, the present investigation considers fundamental aspects of the chemical thermodynamics, transport phenomena in the gas phase, and chemical kinetics of the deposition reaction.

In recent years, various theories have been proposed to describe the transport phenomena in CVD reactors. ${ }^{10-18}$ Most of these have modeled the epitaxial growth of $\mathrm{Si}$ and SiGe layers, and they can be divided into two main groups. The first covers models that were developed on the basis of the boundary-layer theory and only consider physical diffusion effects (e.g., Refs. 10 and 11). The second encompasses models that consider the surface reactions (e.g., Refs. 12-18). Since in CVD reactors, the boundary layer is stationary or at most moving slowly, the input value of partial pressure of the reactants cannot be upheld throughout the chamber. Moreover, the design of the chamber (the height and width of chamber, the size of the susceptor, etc.) can also have an influence on the deposition and the final growth rate of the layer. For modeling that is detailed enough to be predictive, it is necessary to consider both physical phenomena and reaction processes in relationship to the specific reactor design. The lack of modeling that takes both aspects into account has meant that it has been necessary to empirically calibrate each type of reactor with respect to deposition rate.

In this paper, a detailed kinetic model is presented. In addition to theoretical considerations, two different reactor types are experimentally compared, and the model is shown to be potent enough to predict the rate of pure-boron deposition from diborane $\left(\mathrm{B}_{2} \mathrm{H}_{6}\right)$ on a non-rotating silicon wafer. The model takes into consideration the gas-phase diffusion 
mechanism of the diborane species through the stationary boundary layer over the wafer, the gas phase processes and the related surface reactions by applying the actual parabolic gas velocity and temperature gradient profiles in the reactor which were calculated theoretically and also simulated with commercial FLUENT ${ }^{\circledR}$ software.

\section{ANALYTICAL KINETIC MODEL}

In the following sections, derivation of the analytical kinetic model will be discussed in detail.

\section{A. Definition of the reactor system}

In Fig. 1, a schematic illustration is shown of the chemical and physical CVD chamber geometry that we have used in our model. The experimental work on which the modeling is based was performed in two different systems, the ASM Epsilon One and 2000, that have two different reactor designs. In the modeling reactor, the height, $h$, is constant and approximately equal to $22 \mathrm{~mm}$ and the width, $b$, is much larger than $h$. The aspect ratio, $h / b \approx 0.085$, is therefore so small that we can consider this reactor to be a twodimensional system with sufficient accuracy. In these systems, the susceptor lies at the bottom of the chamber and it is heated up and held at deposition temperature, $T_{s}$, through an assembly of lamps. This heat is transferred to the gas flowing over the susceptor, whereas the temperature of the upper wall $\left(T_{w}\right)$ is much cooler than the susceptor and we have assumed its temperature to be $T_{0}$. Moreover, we have assumed that the inlet temperature of the gases is room temperature. Therefore, a large temperature gradient exists over the height of the reactor. This is visualized by FLUENT ${ }^{\circledR}$ simulations in Sec. II B.

The deposition conditions are chosen for which the reactant $\left(\mathrm{B}_{2} \mathrm{H}_{6}\right)$ concentrations can be assumed to be much smaller than the concentration of the carrier gas $\left(\mathrm{H}_{2}\right)$. From this, it follows that the gas flow and temperature profiles are completely determined by the physical constants of the carrier gas. The overall flow velocity and temperature gradients are chosen in such a way that the Reynolds number, $R e$, satisfies the condition for laminar flow in the reactor, ${ }^{19}$ but at the same time, the velocity is chosen just high enough for the transport in the flow direction to be completely dominated by forced flow, i.e., we neglect diffusion along the axes of the reactor system. These effects will, however, be discussed in connection with the experimental results presented in Sec. IV. End effects in flow and temperature are not taken into account so the profiles of the velocity and temperature are fully developed.

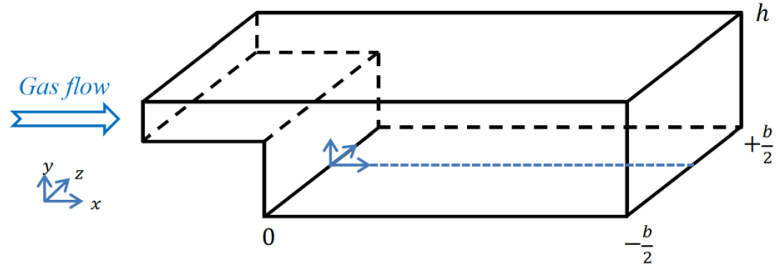

FIG. 1. Schematic illustration of the CVD reactor geometry used for modelling purposes.
Starting point for developing a mathematical model for the chemical and physical behavior of the CVD process is an expression for the temperature, $T$, and velocity profile for a fully developed flow which can be calculated by solving the equation for energy conservation, the continuity equation, and the equation of motion for the carrier gas:

$$
\begin{gathered}
c_{g} \rho_{g} u_{x} \frac{\partial T}{\partial x}=\frac{\partial}{\partial x}\left(k_{0} T^{\beta} \frac{\partial T}{\partial x}\right)=0, \\
\frac{\partial}{\partial y}\left(\mu_{0} T^{\gamma} \frac{\partial u_{x}}{\partial y}\right)=\text { constant }
\end{gathered}
$$

where $u_{x}(y), x, y, c_{g}, \rho_{g}, \mu_{0} T^{\gamma}$, and $k_{0} T^{\beta}$ are the gas velocity profile, axial position in the direction of the gas flow, vertical position perpendicular to the direction of the gas flow, heat capacity of the carrier gas at constant pressure, density of the carrier gas, temperature-dependent thermal conductivity, and dynamic viscosity of the carrier gas, respectively. The constants $\beta$ and $\gamma$ are approximately equal to $0.7 .^{20,21}$

The solution of these equations leads to a distorted parabola with the maximum shifted towards the colder part of the reactor for the gas velocity profile, $u_{x}(y)$, and the gradually decreasing temperature above the susceptor for the temperature profile, $T(y)$, Eq. (3), which are confirmed by simulation in Sec. III A.

$$
T(y)=\left[T_{s}^{1+\beta}-\left(T_{s}^{1+\beta}-T_{s}^{1+\beta}\right) \frac{y}{h}\right]^{\frac{1}{1+\beta}} .
$$

Consequently, the velocity of gas molecules deviates at the gas boundaries where there is a vertical diffusion of the gas molecules. This effect was also considered in the implementation of the model. Lastly, we assume that adsorption of the gas-phase species only occurs on the substrate placed on the hot susceptor and not on the cold upper wall of the reactor. A balance between adsorption and subsequent deposition is established to produce the PureB-layer and/or doping of the substrate.

\section{B. Concentration profile model for diffusion controlled growth rates in a laminar flow system}

In this section, a model for the concentration profile of the reactant species in the horizontal chamber of the ASM Epsilon One CVD reactor is developed. In this case, the deposition process is limited by mass-transport in the gas phase. The main idea of the model is to develop the concentration profile by taking into account the diffusion mechanism of the diborane species through the stationary boundary layer over the wafer and by applying the actual parabolic gas velocity and temperature gradient profiles inside the 2-D reactor system.

In the following treatments, the gas flow is considered to be laminar and dominated by forced convection. This implies that intermingling due to free convection can be neglected. Return flow of heated gas may occur at the leading edge of the hot susceptor. ${ }^{22}$ This is due to buoyancy forces caused by the expansion of the cold gases hitting hot 
elements. This effect, which introduces additional mixing and memory effects, is also neglected in the present model.

\section{Isothermal system}

First, we consider a system with a constant temperature in which the active component rapidly decomposes at the susceptor, $y=0$, for all axial flow positions $x \geq 0$ (Figs. 1 and 2). Therefore, the concentration at $y=0$ is zero across the whole decomposition zone. Transport of material towards the susceptor in the y-direction goes entirely via gas-phase diffusion (laminar flow through the stationary boundary layer over the susceptor).

The equation for mass conservation for this case is

$$
u(y) \frac{\partial C(x, y)}{\partial x}=D \frac{\partial^{2} C(x, y)}{\partial y^{2}},
$$

where $\mathrm{C}$ and $\mathrm{D}$ are the concentration profiles and the gasphase diffusion coefficient of the active component in the carrier gas, respectively, and $u(y)$ is an expression for the parabolic velocity profile found by solving Eqs. (1) and (2) in the reactor chamber and given by

$$
u(y)=4 u_{0}\left(\frac{y}{h}-\frac{y^{2}}{h^{2}}\right) .
$$

Equation (4) is difficult to solve for a parabolic velocity profile. Therefore, the problem is first to be solved for a constant flow velocity, thereafter the influence of a parabolic flow profile on the obtained results is evaluated. For the case of a constant flow velocity $\left(u_{0}\right)$, the problem can be solved analytically for the boundary conditions:

$$
\begin{cases}C(x, 0)=0, & \text { for all } x \geq 0 \\ \left.\frac{\partial C(x, y)}{\partial y}\right|_{y=h}=0, & \text { for all } x \geq 0 \\ C(0, y)=C_{0}, & \text { for all } 0<y \leq h .\end{cases}
$$

The second condition, Eq. (6b), is an expression of the fact that no mass-transport is possible through the upper wall of the reactor. Following the Ref. 23, the above problem can be solved, giving

$$
\begin{aligned}
C(x, y)= & \frac{4 C_{0}}{\pi} \sum_{m=0}^{\infty}\left\{\frac{1}{(2 m+1)} \sin \left(\frac{(2 m+1) \pi}{2 h} y\right)\right. \\
& \left.\times \exp \left[\frac{-(2 m+1)^{2} \pi^{2} D}{4 h^{2} u_{0}} x\right]\right\}
\end{aligned}
$$

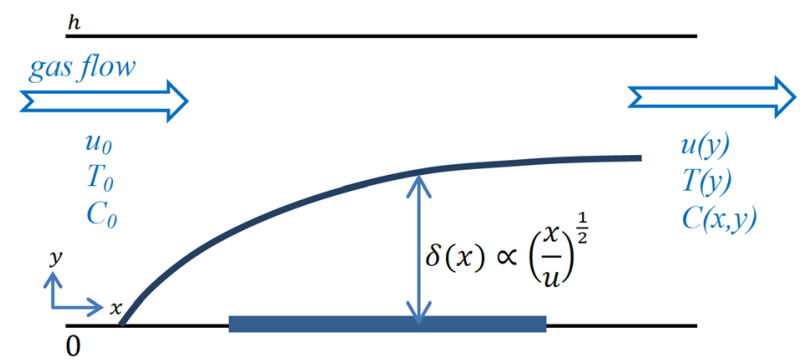

FIG. 2. Schematic illustration of a clasical boundary layer and reactor conditions over the susceptor.

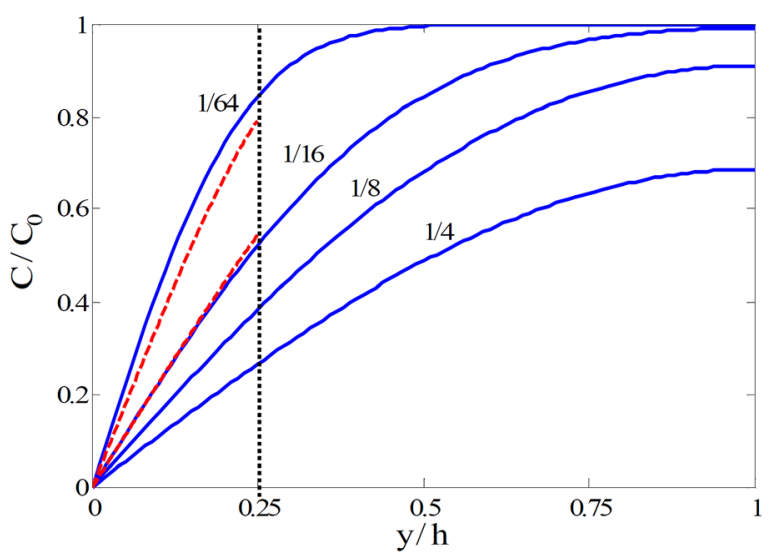

FIG. 3. Normalized concentrations calculated as a function of the axial position $x$ in units of $\frac{h^{2} u_{0}}{D}$, which is indicated along each curve. Solid line: concentration as a function of position above the susceptor in an isothermal cell with a capturing boundary at $y=0$, for a constant gas velocity and temperature. Dashed line: concentration profile for $x_{0}=\frac{h^{2} u_{0}}{64 D}$ and $x_{0}=\frac{h^{2} u_{0}}{16 D}$ after correction for the linear velocity profile. Parameter of the figure is the axial position, $x$ in $\frac{h^{2} u_{0}}{D}$.

In this equation, $m$ is a summation integer.

In Fig. 3, concentration profiles at different positions $x$, i.e., $h^{2} u_{0} / D$, are drawn as calculated from Eq. (7). For small values of $x$, the solution of Eq. (7) is equivalent to an error function for diffusion in a semi-infinite medium. This holds until the concentration at $y=h$ falls significantly below $C_{0}$. For the following analysis, the critical concentration for this to happen is defined as $0.99 C_{0}$ (see Fig. 4). The axial distance $x_{0}$ at which the concentration at $y=h$ reaches this value can be calculated from Eq. (7) as

$$
x_{0}=\frac{h^{2} u_{0}}{16 D} .
$$

It appears that for higher values of $x$, only the first term in the summation of Eq. (7) has to be considered, as the series converges very rapidly. For these values of $x>x_{0}$

$$
C(x, y)=\frac{4 C_{0}}{\pi} \sin \left(\frac{\pi y}{2 h}\right) \exp \left(\frac{-\pi^{2} D}{4 h^{2} u_{0}} x\right) .
$$

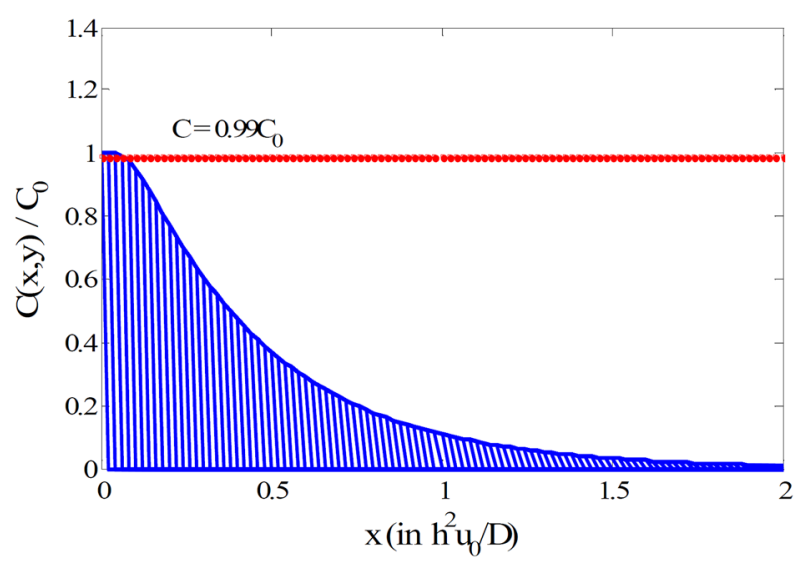

FIG. 4. Normalized concentration found from Eq. (7) versus axial position, $x$ in $\frac{h^{2} u_{0}}{D}$. The entrance length, $x_{0}$, where the $C(x, y)=0.99 C_{0}$, is also indicated. 
In this equation, the gas phase depletion in the flow direction is represented by the exponential term. Equation (9) also shows that the concentration profiles as a function of $y$ have the same form for all values of $x>x_{0}$, as determined by the sine function. Therefore, the distance $x_{0}$ can be considered as an entrance length for the concentration profile to develop in the reactor cell: for $x \leq x_{0}$, the concentration profile has to build up and is the same as in a semi-infinite medium; for $x>x_{0}$, the concentration profile has developed and remains of the same form for all $x$.

\section{Solutions for the development region $x \leq x_{0}$ in a linear flow profile}

Fig. 3 shows that in the development region $x \leq x_{0}$, the largest drop in concentration occurs in a relatively thin layer above the susceptor. Therefore, for calculation of the mass flux at $y=0$, this part of the reactor represents the region of main interest. In Fig. 3, it can be seen that the main concentration drop occurs between $y=0$ and $y=h / 4$. In order to solve Eq. (4) in a more realistic way than the plug velocity approach given above, the parabolic velocity profile can be substituted by a linear velocity distribution as

$$
u(y)=3.4 u_{0}\left(\frac{y}{h}\right) .
$$

This is on the average correct to within $\sim 10 \%$ in the relevant range $0 \leq y \leq h / 4$ as illustrated by Fig. 5 .

Following Ref. 24, the solution for a diffusion problem in a semi-infinite system with a capturing boundary at $y=0$, this linear velocity profile is given by

$$
\frac{C(x, y)}{C_{0}}=1-\frac{\int_{\beta}^{\infty} \exp \left(-\theta^{3}\right) d \theta}{\Gamma\left(\frac{4}{3}\right)},
$$

where

$$
\beta=y\left(0.38 \frac{u_{0}}{D h x}\right)^{1 / 3}
$$

Here, $\theta$ is an integration parameter and $\Gamma$ is the well-known gamma function. The concentration profiles given by

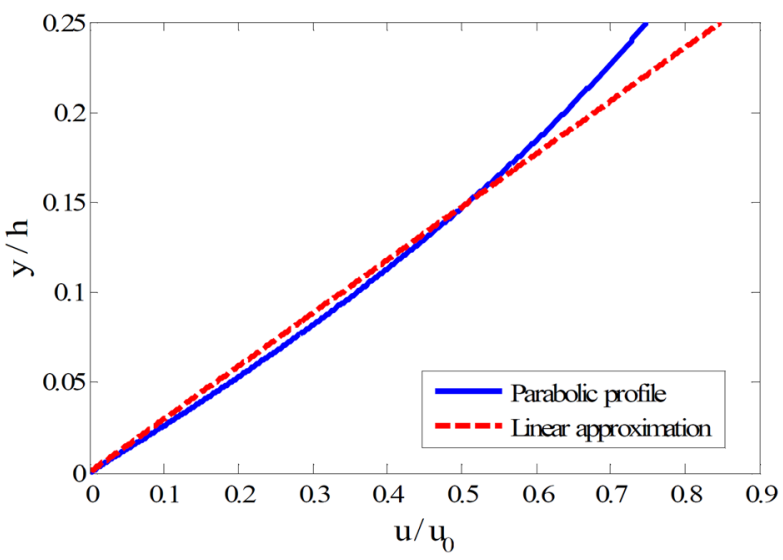

FIG. 5. Parabolic flow profile for $x_{0}=\frac{h^{2} u_{0}}{16 D}$ (solid line) and linear velocity approximation (dashed line) as a function of $y$ for $0 \leq y \leq h / 4$.
Eq. (11a) do not differ strongly from those given by Eq. (7), but can be regarded as a refinement of the model. This is illustrated in Fig. 3 for $x_{0}=\frac{h^{2} u_{0}}{64 D}$ and $x_{0}=\frac{h^{2} u_{0}}{16 D}$ in the interval $0 \leq y \leq h / 4$. Using Fick's law, the mass flux at $y=0$, as given by:

$$
F_{m}(x)=-D\left[\frac{\partial C(x, y)}{\partial y}\right]_{y=0} .
$$

By using Eq. (11a), this now becomes

$$
F_{m}(x)=0.81 D C_{0}\left(\frac{D h x}{u_{0}}\right)^{-1 / 3}
$$

As mentioned in Ref. 8 and by classical boundary layer theory, which equate for the stationary boundary layer and mass flux over the susceptor, $F_{m}(x)=D C_{0} / \delta(x)$ and $\delta(x)=\left(\frac{\mu x}{\rho u}\right)^{\frac{1}{2}}$, respectively, the factor $\frac{1}{0.81}\left(\frac{D h x}{u_{0}}\right)^{\frac{1}{3}}$ in Eq. (12b) can be considered as the effective boundary layer thickness, $\delta_{e f f}(x)$. The error in the mass flux given in Eq. (12b) introduced by the linear velocity profile is small $(\sim 3 \%)$ due to the power of $1 / 3$ in $u_{0}$. The mass flux follows a $1 / 3$ power in $x$ and $u$ as a consequence of the linear velocity approximation.

Because the active component is only consumed at $y=0$ (over the susceptor), the average concentration at $x=x_{0}$, $\bar{C}\left(x_{0}\right)$, can now be calculated on the basis of the total amount of material that has disappeared as follows:

$$
\int_{0}^{x_{0}} F_{m}(x) d x=h u_{0}\left[C_{0}-\bar{C}\left(x_{0}\right)\right] .
$$

Solving this equation gives

$$
\bar{C}\left(x_{0}\right)=0.81 C_{0} .
$$

Using this and Eqs. (8) and (12b), we obtain for the mass flux at $x=x_{0}$ and $y=0$ :

$$
F_{m}\left(x_{0}\right)=2.52 \frac{D \bar{C}\left(x_{0}\right)}{h} .
$$

\section{Solutions for the depletion region $x>x_{0}$}

Since the concentration profiles for values of $x$ larger than the entrance length are of the same form (this will remain true for any velocity function which is constant in $x$ ), Eq. (14) can be generalized by applying symmetry considerations:

$$
F_{m}(x)=2.52 \frac{D \bar{C}(x)}{h} .
$$

The mass flux balance between the supply by forced flow and deposition at $y=0$ for each $x$ is now given by

$$
-h u_{0} \frac{\partial \bar{C}(x)}{\partial x}=2.52 \frac{\bar{C}(x)}{h} .
$$

Integration of this equation with respect to $x$ from $x_{0}$ to $x$ and with respect to $C(x)$ from $0.81 C_{0}$ to $C(x)$ gives 


$$
\bar{C}(x)=0.81 C_{0} \exp \left[\frac{-2.52 D\left(x-x_{0}\right)}{h^{2} u_{0}}\right] .
$$

Then the concentration profiles at $y=0$ can be calculated from Eq. (17). After rearranging, using Eq. (8)

$$
\bar{C}(x)=0.692 C_{0} \exp \left[\frac{-2.52 D}{h^{2} u_{0}} x\right] .
$$

This is the average concentration profile of the reactants over the susceptor with linear velocity profile. This will be used for developing the final deposition rate model for PureB layer deposition.

\section{Modeling of the active precursor flux based on gas phase processes and related surface reactions}

For a gas-solid heterogeneous unimolecular elementary reaction, there are two essential conditions needed for reactant molecules to deposit onto the substrate.

(i) The reactant molecules must be activated.

(ii) The reactant molecules must interact with the substrate.

The Meng Tao method of modeling the growth rate of the CVD of Si from silane ${ }^{14}$ is presented here as a basis for the approach taken for the PureB case. In Tao's model, the impinging reactant molecules on the Si surface are attached to the dangling bonds. By applying the Maxwell distribution function in unit time, the number of the reactant molecules $(\mathrm{d} \Gamma)$ which interact with a unit area of the substrate with kinetic energy between $E_{K}$ and $\left(E_{K}+d_{K}\right)$ can be thus estimated to be

$$
d \Gamma=8 \pi N_{R}\left(\frac{1}{2 \pi m_{R} k_{b} T}\right)^{\frac{3}{2}} m_{R} E_{k} \exp \left[-\frac{E_{k}}{k_{b} T}\right] d E_{k},
$$

where $N_{R}$ is the number of reactant molecules in a unit volume of the gas phase, $m_{R}$ is the mass of a reactant molecule, and the $k_{b}$ is Boltzmann's constant at deposition temperature. Integrating the formula from $E_{A}$ (deposition activation energy) to $+\infty$, the number of the activated reactant molecules which strike a unit area of the substrate in one unit of time is given by

$$
\Gamma=\frac{N_{R}}{\left(2 \pi m_{R} k_{b} T\right)^{\frac{1}{2}}}\left(E_{A}+k_{b} T\right) \exp \left(-\frac{E_{A}}{k_{b} T}\right) .
$$

So the activated flux of a precursor, i.e., the number of precursor species that decomposes upon collision with the substrate is

$$
f_{R}^{S}=\frac{P_{R}^{S}}{\left(2 \pi m_{R} k_{b} T\right)^{\frac{1}{2}}}\left(\frac{E_{A}}{k_{b} T}+1\right) \exp \left(-\frac{E_{A}}{k_{b} T}\right),
$$

where $P_{R}^{S}$ is the partial pressure of the reactant at the surface of the substrate and $E_{A}$ is the activation energy of the heterogeneous reaction, and assuming a perfect gas for which $P=N k T$. Although several studies have been published on reactions between $\mathrm{B}$ hydrides and $\mathrm{Si} / \mathrm{PureB}$ surfaces ${ }^{25-28}$ ) little is known about the reaction mechanism of diborane, $\mathrm{B}_{2} \mathrm{H}_{6}$, on the Si/PureB surfaces. In the gas phase, $\mathrm{B}_{2} \mathrm{H}_{6}$ undergoes homogeneous decomposition ${ }^{29,30}$ ) by reaction R1:

$$
\text { [R1] } \mathrm{B}_{2} \mathrm{H}_{6}(\mathrm{~g}) \rightarrow 2 \mathrm{BH}_{3}(\mathrm{~g}) \text {. }
$$

Following the Ref. $31, \mathrm{BH}_{3}$ adsorption brings $\mathrm{H}$ to the surface, which affects the surface $\mathrm{H}$ coverage and hence the deposition rate. With one precursor involved and four types of surface sites, $\mathrm{H}$-terminated $\mathrm{Si}$ and $\mathrm{B}$ sites and $\mathrm{H}$-free $\mathrm{Si}$ and $\mathrm{B}$ sites, there are four heterogeneous reactions to consider, which are listed in Table I. In Fig. 6, an illustration is given of these reactions.

Then the total B-flux over the substrate surface can be expressed as the sum of the above mentioned four fluxes of reactions [R2] to [R5].

On the other hand, the activation energies for reactions on $\mathrm{H}$-terminated $\mathrm{Si} / \mathrm{PureB}$ surface sites are larger than $\mathrm{H}$-free sites. Thus, these fluxes can be neglected as compared to those on $\mathrm{H}$-free $\mathrm{Si} / \mathrm{PureB}$ surface sites. As an example, the activation energy of $\mathrm{SiH}_{4}$ adsorption on $\mathrm{H}$-free $\mathrm{Si}$ sites is $\sim 30 \mathrm{kcal} /$ $\mathrm{mol},{ }^{32}$ whereas that on H-terminated $\mathrm{Si}$ sites is $\sim 51 \mathrm{kcal} /$ $\mathrm{mol}^{33}$ and it is reasonable to assume that the situation for the deposition of $\mathrm{BH}_{3}$ would show a similar trend. Under this assumption, the total surface B flux can be expressed as

$$
\begin{aligned}
f_{B}^{S} & =f_{B H_{3}-o n-S i}^{S}+f_{B H_{3}-o n-H S i}^{S}+f_{B H_{3}-o n-B}^{S}+f_{B H_{3}-o n-H B}^{S} \\
& \approx f_{B H_{3}-o n-S i}^{S}+f_{B H_{3}-o n-B}^{S} .
\end{aligned}
$$

Based on Eq. (21), we can write down the equation for the primary surface B flux as

$$
\begin{aligned}
f_{B}^{S} \approx & \eta_{1}(1-\gamma)\left(1-\theta_{H(S i)}\right) \frac{P_{B H_{3}}^{S}}{\left(2 \pi m_{B H_{3}} k_{b} T\right)^{\frac{1}{2}}}\left(\frac{E_{B H_{3}-o n-S i}}{k_{b} T}+1\right) \\
& \times \exp \left(-\frac{E_{B H_{3}-o n-S i}}{k_{b} T}\right) \\
& +\eta_{2} \gamma\left(1-\theta_{H(B)}\right) \frac{P_{B H_{3}}^{S}}{\left(2 \pi m_{B H_{3}} k_{b} T\right)^{\frac{1}{2}}}\left(\frac{E_{B H_{3}-o n-B}}{k_{b} T}+1\right) \\
& \times \exp \left(-\frac{E_{B H_{3}-o n-B}}{k_{b} T}\right)
\end{aligned}
$$

TABLE I. Heterogeneous reaction possibilities involved in PureB-layer CVD with $\mathrm{B}_{2} \mathrm{H}_{6}$ as a precursor.

\begin{tabular}{lccc}
\hline \hline Reaction no. & Notation & $\mathrm{BH}_{3}$ reaction with & Reaction \\
\hline$[\mathrm{R} 2]$ & $f_{B H_{3}-o n-S i}$ & H-free Si surface sites & $B H_{3}(g)+\cdot \boldsymbol{S i}(s) \rightarrow H_{2} B \_S i(s)+H(g)$ \\
{$[\mathrm{R} 3]$} & $f_{B H_{3}-o n-H S i}$ & H-terminated Si surface sites & $B H_{3}(g)+H_{-} S i(s) \rightarrow H_{2} B \_S i(s)+H_{2}(g)$ \\
{$[R 4]$} & $f_{B H_{3}-o n-B}$ & H-free PureB surface sites & $B H_{3}(g)+\bullet B(s) \rightarrow H_{2} B B(s)+H(g)$ \\
{$[$ R5] } & $f_{B H_{3}-o n-H B}$ & H-terminated PureB surface sites & $B H_{3}(g)+H B(s) \rightarrow H_{2} B \_B(s)+H_{2}(g)$ \\
\hline \hline
\end{tabular}




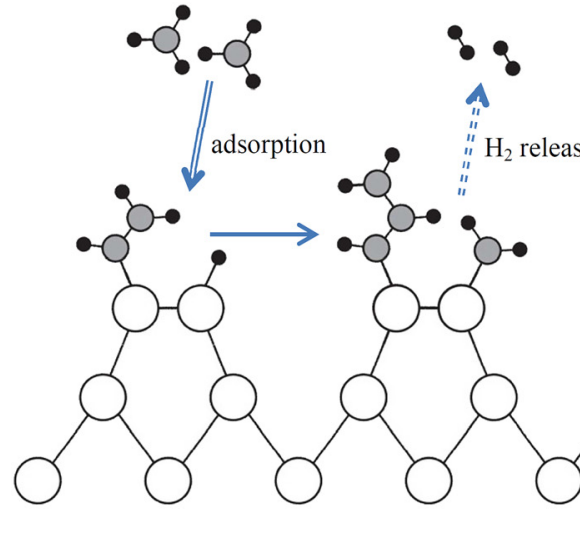

H-terminated sites

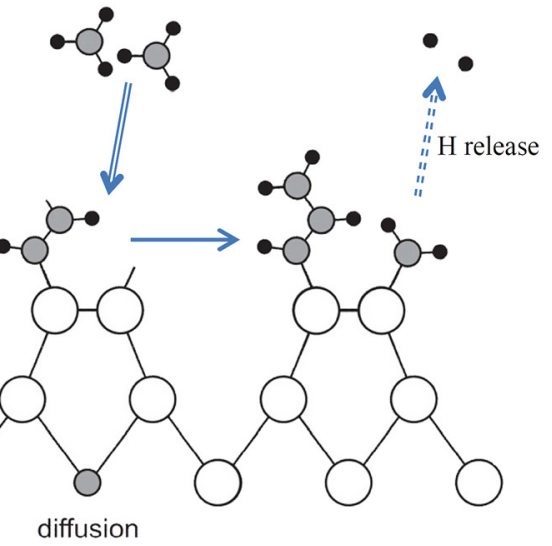

H-free sites

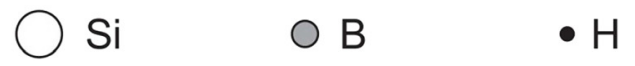

FIG. 6. Heterogeneous reaction possibilities involved in PureB-layer CVD with $\mathrm{B}_{2} \mathrm{H}_{6}$ as a precursor.
In these equations, $\theta_{H(S i)}$ and $\theta_{H(B)}$ are the ratio of $\mathrm{H}$ terminated $\mathrm{Si}$ sites to all $\mathrm{Si}$ sites and the ratio of $\mathrm{H}$ terminated PureB surface sites to all PureB surface sites, respectively. The $P_{B H_{3}}^{S}$ and $m_{\mathrm{BH}_{3}}$ are the $\mathrm{BH}_{3}$ partial pressure at the substrate surface and molecular mass of $\mathrm{BH}_{3}$, respectively. The $E_{B_{3}-o n-S i}$ and $E_{B H_{3}-o n-B}$ are the activation energies unique to the particular reaction ([R2], [R4]). The $\gamma$ is the ratio of PureB surface sites to all surface sites and is calculated as $[\mathrm{B}] / \mathrm{N}_{0}$, where $[\mathrm{B}]$ is the $\mathrm{B}$ concentration and $\mathrm{N}_{0}$ the atomic density of the PureB-layer, which is $5.0 \times 10^{22}$ atoms $/ \mathrm{cm}^{3}$ at Si surfaces and $1.3 \times 10^{23}$ atoms $/ \mathrm{cm}^{3}$ at PureB surfaces. ${ }^{34}$ Also $\eta$ is a unit less constant.

As mentioned in Ref. 7, at the early stages of deposition, such as sub-min deposition times, boron atoms are deposited and interact to cover the silicon surface with an atomic-layer plane and the boron coverage can also grow to exceed one monolayer (1 ML). Thus the PureB deposition can be divided into the two stages. In the first stage, a monolayer coverage of pure-boron atoms on the Si surface is being built up and in the second stage the pure-boron atoms are deposited on a surface fully covered with boron atoms. Here, only second stage is modeled. Then, the activated boron flux on the PureB surface is reduced to

$$
\begin{aligned}
f_{B}^{S} \approx & f_{B H_{3}-o n-B}^{S} \approx \eta \gamma\left(1-\theta_{H(B)}\right) \frac{P_{B H_{3}}^{S}}{\left(2 \pi m_{B H_{3}} k_{b} T\right)^{\frac{1}{2}}} \\
& \times\left(\frac{E_{B H_{3}-o n-B}}{k_{b} T}+1\right) \exp \left(-\frac{E_{B H_{3}-o n-B}}{k_{b} T}\right) .
\end{aligned}
$$

With Eqs. (18) and (25) and using the ideal gas law in a very thin layer over the susceptor, the final equation for the total activated B flux over the susceptor $(y=0)$ is obtained as

$$
\begin{aligned}
f_{B}(x) \approx & 0.264 \eta \gamma\left(1-\theta_{H(B)}\right) \frac{P_{B H_{3}}}{\left(m_{B H_{3}} k_{b} T\right)^{\frac{1}{2}}}\left(\frac{E_{B H_{3}-o n-B}}{k_{b} T}+1\right) \\
& \times \exp \left(-\frac{E_{B H_{3}-o n-B}}{k_{b} T}\right) \times \exp \left(-\frac{2.52 D}{h^{2} u_{0}} x\right) .
\end{aligned}
$$

In this equation, the $P_{B H_{3}}$ is the input partial pressure of the $\mathrm{BH}_{3}$ and the $E_{\mathrm{BH}_{3}-\mathrm{On}-\mathrm{B}}$ is the unique activation energy of the PureB CVD. ${ }^{31}$
If the density of the deposited PureB-layer is $\rho_{B}$ and the mass of the pure-boron atoms in the layer is $\mathrm{M}$, the number of boron atoms in a unit volume of the PureB-layer is

$$
N_{0}=\frac{\rho_{B}}{M}
$$

From the equations given above, the deposition rate, $D R$, of the PureB-layer deposited in a CVD system by using $\mathrm{B}_{2} \mathrm{H}_{6}$ can be calculated as

$$
\begin{aligned}
D R(x)= & \frac{f_{B}(x)}{N_{0}} \\
= & 0.264 \eta \gamma \frac{\left(1-\theta_{H(B)}\right)}{N_{0}} \frac{P_{B H_{3}}}{\left(m_{B H_{3}} k_{b} T\right)^{\frac{1}{2}}}\left(\frac{E_{B H_{3}-o n-B}}{k_{b} T}+1\right) \\
& \times \exp \left(-\frac{E_{B H_{3}-o n-B}}{k_{b} T}\right) \times \exp \left(-\frac{2.52 D}{h^{2} u_{0}} x\right)
\end{aligned}
$$

\section{EXPERIMENTAL}

Experiments were carried out in two systems, the ASM Epsilon One and 2000, that have a quartz reactor with a rectangular cross-section. These reactors have a large SiC susceptor which is heated up to the deposition temperature by a crossed array of lamps above and below of the deposition chamber. The readout and control of the temperature is performed by one master thermo-couple at the center and three slaves at the front, rear, and side of the susceptor that was found to be kept at a constant temperature within $\pm 0.5^{\circ} \mathrm{C}$. For deposition of the PureB-layer, diborane was used as a gas source with different input partial pressures from 1.7 to 3.39 mtorr. Pure $\mathrm{H}_{2}$ was used as a carrier gas with a water and oxygen content below the ppm level with variable flow rates between 5 and $20 \mathrm{slm}$ (standard liter per minute). All depositions were performed at $700{ }^{\circ} \mathrm{C}$ and atmospheric pressure (ATM) over $100 \mathrm{~mm}$ non-rotating bare Si (100) wafers with a thickness of 500-550 $\mu \mathrm{m}$. Also we found that the substrate type (orientation, resistivity and doping type) did not influence the deposition rate significantly. 
The PureB deposition is disturbed by any oxide, even few atomic layers of native oxide, ${ }^{7}$ and any particle contamination or residues on the surface. Therefore, before loading into the reactor, samples were immersed in a diluted HF $(0.55 \%)$ solution for $4 \mathrm{~min}$ to remove native oxide and $\mathrm{H}$-passivate the surface against native oxide formation. This is followed by Marangoni drying, which is an effective substitute for spin rinse drying as the formation of drying spots is avoided. These otherwise readily form on mixed hydrophilic-hydrophobic surfaces and particle contamination results from residues left behind after evaporation. ${ }^{39}$ After drying, the samples are immediately put in the load-lock of the reactor where they are continuously purged by oxygenfree $\mathrm{N}_{2}$ gas. As an extra measure to assure an oxygen-free surface, a $4 \mathrm{~min} \mathrm{H}$-bake at $800^{\circ} \mathrm{C}$ was performed before the deposition. ${ }^{7}$ The layer thickness was measured in-line using ellipsometry which has an acceptable accuracy and good repeatability for smooth layers. ${ }^{8}$ The inlet region before the deposition zone (see Fig. 1) is around $15 \mathrm{~cm}$ for our reactors, which was calculated to be long enough to allow the velocity profile to be established. ${ }^{35}$ The hot zone suited for reaction/ deposition is $25 \mathrm{~cm}$ in length. For the experimental data, the position $x=0$ defines the leading edge of the wafer on the hot susceptor.

\section{A. Application of the theoretical model to the CVD process}

It is a generally accepted fact that the CVD of boron using diborane with hydrogen carrier gas is limited by gas phase diffusion of $\mathrm{BH}_{3}$ molecules through the stationary boundary layer over the wafer (e.g., Ref. 40). Diborane is known to decompose in $\mathrm{H}_{2}$ at temperatures above 200 $300{ }^{\circ} \mathrm{C}^{29}$ Also it can be observed from Fig. 7 that there is only in a narrow region close to the susceptor where the gases are heated to the deposition temperature and therefore the assumption that $\mathrm{B}_{2} \mathrm{H}_{6}$ molecules decompose just over the susceptor is acceptable. The upper wall of the chamber is not

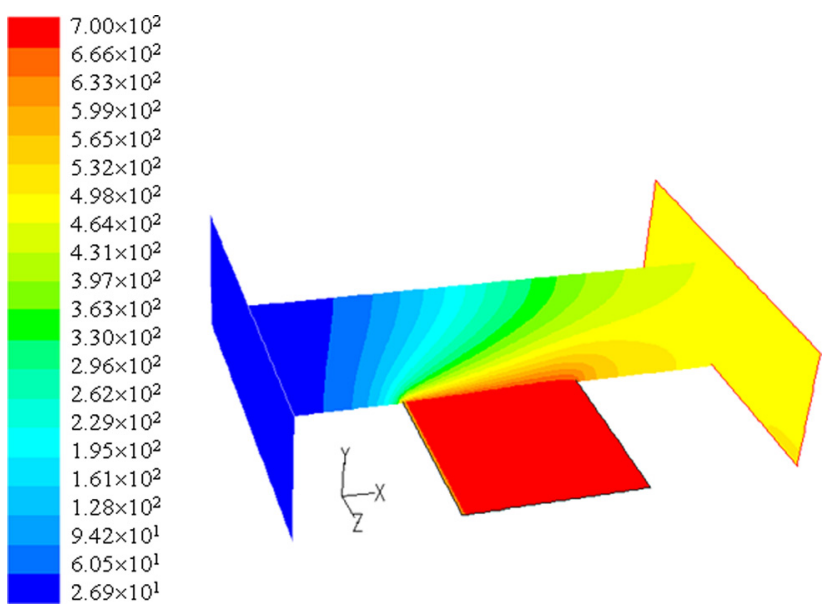

FIG. 7. The temperature distribution for an ASM Epsilon One CVD reactor as simulated by commercial FLUENT software with the total pressure at ATM. To simplify the simulation, hydrogen is considered to be the main gas flowing over the susceptor. The susceptor is heated up by an assembly of lamps to the deposition temperature (here $700{ }^{\circ} \mathrm{C}$ ) and this heat is transferred to the flowing gas. heated with lamps, so the decomposition will not take place at this cold wall (see Fig. 7).

From the above, it can be concluded that under forced laminar flow conditions, the theoretical model discussed in Sec. II C should be applicable to the present systems. As far as we know, no measurements are available for the diffusivity of $\mathrm{BH}_{3}$ in $\mathrm{H}_{2}$, so in the present analysis, the diffusion coefficients for the undecomposed $\mathrm{BH}_{3}$ molecule are used. From Ref. 36, it can be found that the diffusion coefficient for $\mathrm{BH}_{3}$ with $\mathrm{M}=13.84 \mathrm{~g} / \mathrm{mol}$ is approximately $0.77 \mathrm{~cm}^{2} / \mathrm{s}$ at $273 \mathrm{~K}$ and $1 \mathrm{~atm}$ in $\mathrm{H}_{2}$. Then following this reference, this value can be extrapolated to a temperature of $973 \mathrm{~K}$ $\left(\sim 700^{\circ} \mathrm{C}\right)$ by using this empirical equation:

$$
\frac{D_{B H_{3}}^{T_{1}}}{D_{B H_{3}}^{T_{2}}}=\left[\frac{1}{2}\left(\frac{T_{1}}{T_{2}}+1\right)\right]^{1.65} .
$$

Then the physical constant for $\mathrm{BH}_{3}$ in $\mathrm{H}_{2}$ at $1 \mathrm{~atm}$ and $700{ }^{\circ} \mathrm{C}$ is

$$
D_{B H_{3}}^{700}{ }^{\circ}\left(H_{2}\right)=3 \frac{\mathrm{cm}^{2}}{\mathrm{~s}} \text {. }
$$

Since these diffusion coefficients vary approximately with the square root of the reduced molecular mass of the diffusing molecules, only small differences for the diffusion of the various possible diborane species are expected.

Recently, we reported in Ref. 31, the activation energies of the reaction/deposition of the PureB-layer. It is found to be $6.5 \mathrm{kcal}^{\mathrm{mol}}{ }^{-1}$ for moderate temperatures between $400^{\circ} \mathrm{C}$ and $700^{\circ} \mathrm{C}$ and $28 \mathrm{kcal}^{\mathrm{mol}}{ }^{-1}$ for lower temperatures (lower than $400^{\circ} \mathrm{C}$ ).

\section{RESULTS AND DISCUSSION}

In Table II, the main parameters describing the present experimental conditions are given.

In Fig. 8, several experimental results are compared to model predictions. It should be noted that parameter fitting was performed for the ASM Epsilon One. The PureB deposition rates are extracted as a function of (a) the axial position, $x$, for different gas flow and diborane partial pressure conditions, (b) gas flow over the susceptor, and (c) input diborane partial pressure including curves for two different axial positions. By only adjusting the reactor/process parameters, this model was also successfully transferred from the ASM Epsilon One to the Epsilon 2000 reactor which has totally different reactor conditions. The experimental results and model predictions for the Epsilon 2000 are also shown in Fig. 8(a). In fact, this model has the capability to predict the deposition rate on any 2-D uniform or non-uniformly patterned wafer such as those used for advanced device fabrication. A very

TABLE II. The main parameters describing the experimental conditions for both the Epsilon One and Epsilon 2000.

\begin{tabular}{ccccccc}
\hline \hline EPI & $h(\mathrm{~mm})$ & $h / b$ & $u_{0} \min (\mathrm{slm})$ & $u_{0} \max (\mathrm{slm})$ & $P_{B_{2} H_{6}} \min$ & $P_{B_{2} H_{6}} \max$ \\
\hline One & 22 & 0.085 & 10 & 20 & 1.7 mtorr & 3.39 mtorr \\
2000 & 24 & 0.089 & & & & \\
\hline
\end{tabular}



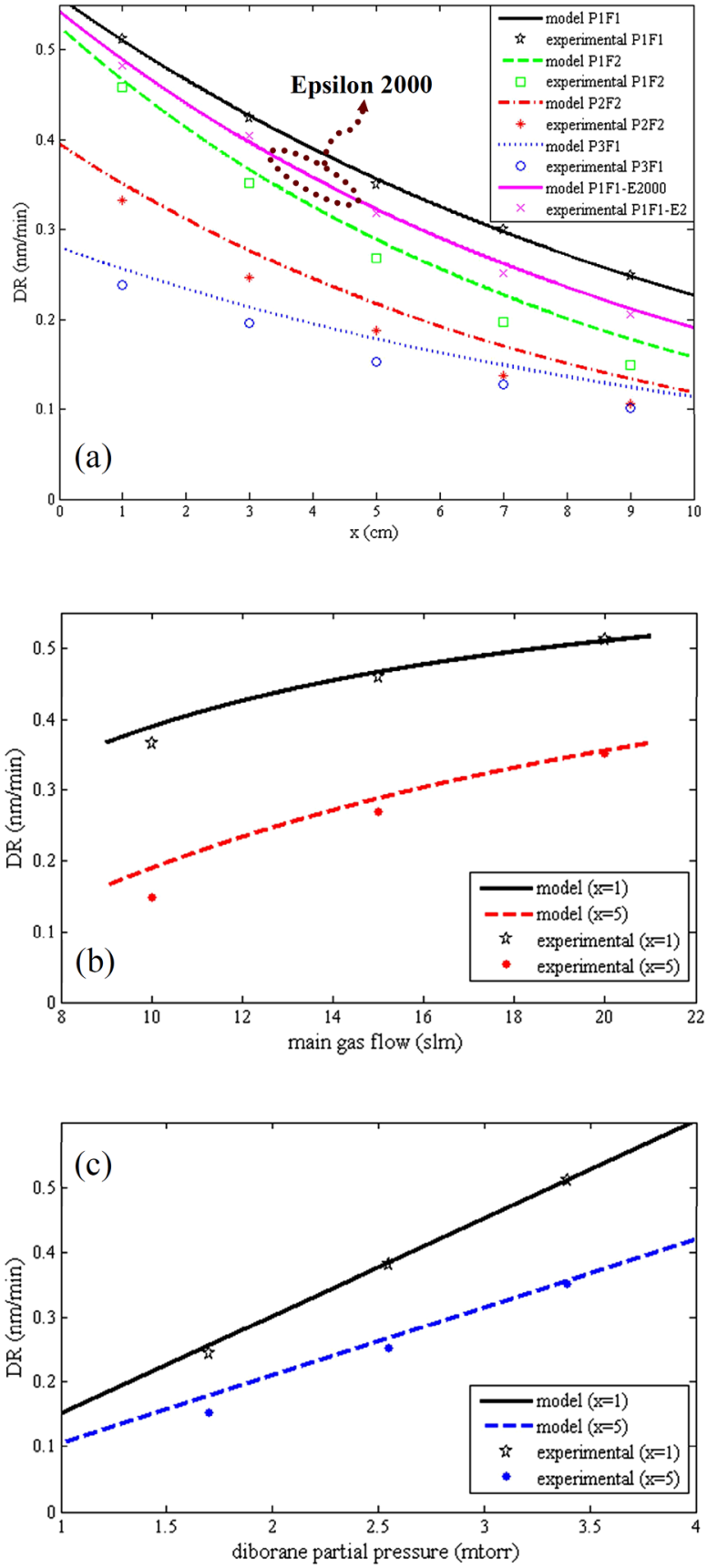

FIG. 8. Model and experimental results for the PureB deposition rate as a function of (a) an axial position, $x$, (b) main gas flow over the susceptor, and (c) diborane partial pressure. The applied diborane partial pressures were $3.39,2.55$, and 1.7 mtorr given by $\mathrm{P}_{1}, \mathrm{P}_{2}$, and $\mathrm{P}_{3}$, respectively. And the applied gas flows were 20,15 , and $10 \mathrm{slm}$ given by $\mathrm{F}_{1}, \mathrm{~F}_{2}$, and $\mathrm{F}_{3}$, respectively. All experiments were performed at atmospheric pressure. small, less than 5\%, deviation between experimental results and model prediction is plausibly related to the lateral diffusion of the diborane molecules and it becomes more evident at lower gas flows and diborane partial pressures.

It can be seen that the data calculated on the basis of this model fits well with the experimental results, and it has been very useful in the development of uniform PureB-layers with little pattern dependence as well as for transferring recipes from one reactor to the other.

\section{A. Remarks on validity of the model for laminar flow and influence of axial and lateral diffusion of diborane species}

The Eq. (28) for the deposition rate in a laminar flow system is, in principle, only valid for the two-dimensional case of two semi-infinite parallel plates. A cell with a rectangular cross-section can also be considered as a twodimensional system if the following requirements are fulfilled. First, the lateral concentration gradients should be small so as to minimize the diffusion in this direction. Second, the gas flow velocity must be constant in the lateral direction. Finally, free convective movements that can cause differences in the supply of reactant components laterally should be unimportant. ${ }^{12}$

To examine this, PureB deposition rates were also studied as a function of the lateral position, $z$, on the wafer. We found that upon decreasing the diborane partial pressure and/ or gas flow rate during the deposition, the axial and lateral homogeneity are disturbed as can be seen in Fig. 9 .

This phenomenon can be attributed to axial and lateral diffusion of the diborane species due to the lateral concentration gradients and lateral gas velocity profile. But in our case, this is not considerable. From this, we infer that lateral diffusion effects will, at least in the deposition region, not play an important role. On the other hand, the gas velocity can be calculated as a function of lateral position, $z$, using an empirical expression derived by Holmes and Vermeulen ${ }^{37}$ as shown in Fig. 10. The results are shown in this figure for the four different rectangular reactor cells with a comparison to the reactor we used in this experiment. The shapes of the velocity profiles strongly depend on the aspect ratio, $h / b$, of the reactor. It can be seen that in our reactor, the gas velocity is constant to within $80 \%$ of the total reactor width. There is a minor influence as can be seen by the deposition rates in Fig. 9 .

Therefore, it is concluded that the lateral distribution of deposition rates is determined by the lateral profile of the

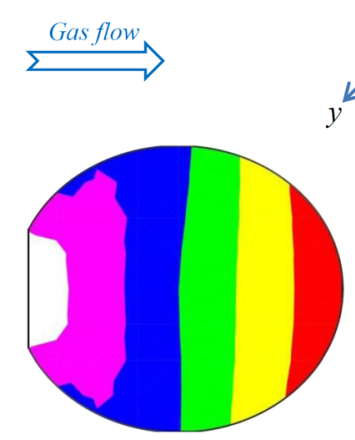

(a)
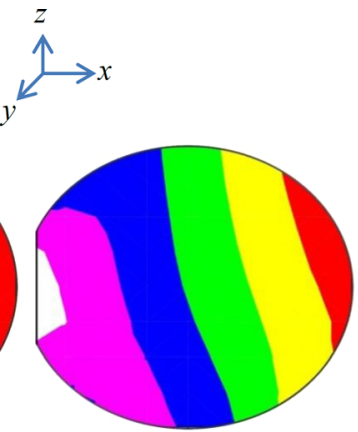

(b)

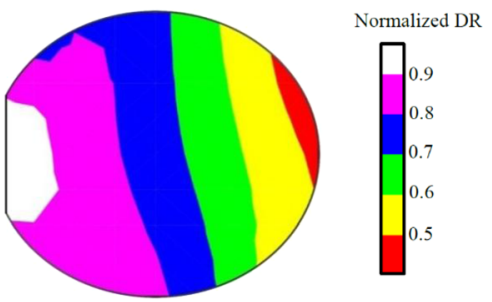

(c)
FIG. 9. 2D contour plots of the normalized PureB deposition rate over a non-rotating wafer for (a) $P_{1} F_{1}$, (b) $P_{1} F_{3}$, and (c) $P_{3} F_{1}$ conditions. Here, $P_{1}$ and $P_{3}$ are 3.39 and 1.7 mtorr, $F_{1}$ and $F_{3}$ are 20 and $10 \mathrm{slm}$, respectively. 


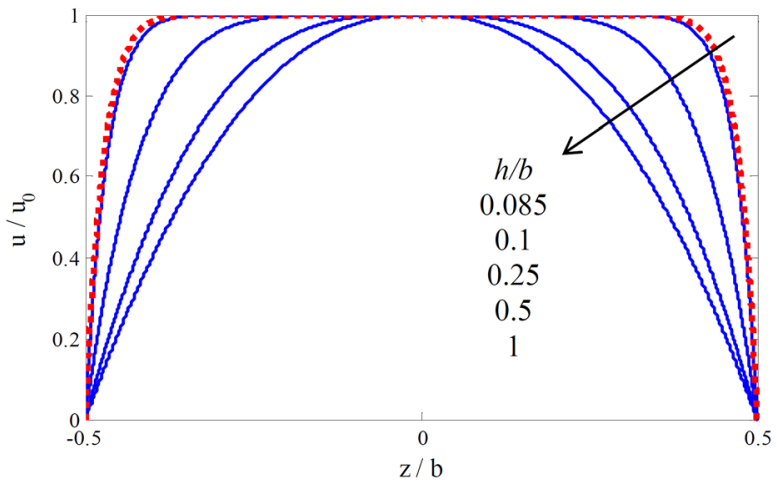

FIG. 10. Lateral distribution of gas velocity at a specific height above the susceptor for the $\mathrm{h} / \mathrm{b}$ ratios $0.085,0.1,0.25,0.5$, and 1 , according to Ref. 37 .

forced flow velocity in these low $R_{a}$ number systems. This implies that free convective motions are not important. Giling's holographic experiments ${ }^{38}$ showed that isotherms are perfectly parallel to the susceptor and gas flow patterns are very stable under the present conditions. From this, it was concluded that in the part of the cell where flow and temperature profiles are developed, free convective motions are not important. From the present work, it can be found that if free-convective motions are present, they do not affect mass transport by diffusion and forced flow under low $R_{a}$ number conditions. Therefore, when considering the deposition rate distributions in our situation, the flow may be assumed to be effectively forced laminar.

\section{CONCLUSIONS}

In this paper, an analytical model was developed to describe the deposition kinetics and the deposition chamber characteristics that determine the deposition rate of PureB layers over the wafer. This model is based on a wide range of input parameters, such as initial diborane partial pressure, total gas flow, axial position on the wafer, deposition temperature, activation energy of diborane deposition, surface $\mathrm{H}$-coverage, and reactor dimensions. By only adjusting these reactor-process parameters, this model was also successfully transferred from the ASM Epsilon One to the Epsilon 2000 reactor which has totally different reactor conditions.

It can be concluded that the data calculated on the basis of this model fits well with the experimental results. A very small, less than 5\%, deviation between experimental results and model prediction is plausibly related to the lateral diffusion of the diborane molecules and it becomes more evident at lower gas flows and diborane partial pressures. The influence of axial and lateral diffusion of diborane species and the validity of the model for laminar flow in the real CVD process was clarified. In fact, this model is capable of predicting the deposition rate on any 2-D uniform or nonuniformly patterned wafer such as those used for advanced device fabrication and it has been very useful in the development of uniform PureB-layers with minimal pattern dependence as well as for transferring recipes from one reactor to the other. As a result, the deposition of 2-nm-thick PureBlayers with only a few angstrom thickness variations is now performed standardly for a variety of process wafer conditions. ${ }^{8}$

\section{ACKNOWLEDGMENTS}

The authors would like to thank A. Sammak and the staff of the DIMES-ICP cleanrooms, particularly T. L. M. Scholtes, for their support in the fabrication and measurement of the experimental material. This work was supported by Project No. 10024 of The Dutch Technology Foundation STW and has profited from collaboration with the SmartMix Memphis project.

${ }^{1}$ F. Sarubbi, L. K. Nanver, and T. L. M. Scholtes, IEEE Trans. Electron Devices 57(6), 1269 (2010).

${ }^{2}$ A. Šakic, L. K. Nanver, G. van Veen, K. Kooijman, P. Vogelsang, T. L. M. Scholtes, W. de Boer, W. H. A. Wien, S. Milosavljevi, C. Th. H. Heerkens, T. Kneževi, and I. Spee, in Proceedings of the International Electron Devices Meeting (IEDM' 10) (2010), pp. 712-713.

${ }^{3}$ L. Shi, F. Sarubbi, L. K. Nanver, U. Kroth, A. Gottwald, and S. Nihtianov, Procedia Eng. 5, 633 (2010).

${ }^{4}$ L. Shi, F. Sarubbi, S. N. Nihtianov, L. K. Nanver, T. L. M. Scholtes, and F. Scholze, in Proceedings of the 35th Annual Conference of IEEE Industrial Electronics Society (IECON) (2009), p. 1877.

${ }^{5}$ L. K. Nanver, in Advances in Photodiodes, edited by Gian Franco and Dalla Betta, ISBN: 978-953-307-163-3 (InTech, 2011), p. 205.

${ }^{6}$ L. K. Nanver, T. L. M. Scholtes, F. Sarubbi, W. B. de Boer, G. Lorito, A. Sakic, S. Milosavljevic, C. Mok, L. Shi, S. Nihtianov, and K. Buisman, in Proceedings of the 18th IEEE Conference on Advanced Thermal Processing of Semiconductors (RTP) (2010), pp. 136-139.

${ }^{7}$ F. Sarubbi, T. L. M. Scholtes, and L. K. Nanver, J. Electron. Mater. 39(2), 162 (2010).

${ }^{8}$ V. Mohammadi, W. B. de Boer, T. L. M. Scholtes, and L. K. Nanver, J. Solid State Sci. Technol. 1(1), Q16 (2012).

${ }^{9}$ D. W. Shaw, Crystal Growth (Plenum, New York, 1974).

${ }^{10}$ F. C. Eversteyn, P. J. W. Severin, C. H. J. van den Brekel, and H. L. Peek, J. Electrochem. Soc. 117, 925 (1970).

${ }^{11}$ L. Vandenbulcke and C. Vuillard, J. Electroehem. Soc. 124, 1931 (1977).

${ }^{12}$ R. Takahashi, Y. Koga, and K. Sugawara, J. Electrochem. Soc. 119, 1406 (1972).

${ }^{13}$ C. W. Manke and L. F. Donaghey, J. Electrochem. Soc. 124, 561 (1977).

${ }^{14}$ M. Tao, Thin Solid Films 223, 201 (1993).

${ }^{15}$ A. V. Potapov, L. K. Orlov, and S. V. Ivin, Thin Solid Films 336, 191 (1998).

${ }^{16}$ B. Mehta and M. Tao, J. Electrochem. Soc. 152, G309 (2005)

${ }^{17}$ X. L. Yang and M. Tao, J. Electrochem. Soc. 154(1), H53 (2007).

${ }^{18}$ M. Kolahdouz, L. Maresca, R. Ghandi, A., Khatibi, and H. Radamson, ECS Trans. 33(6), 581 (2010).

${ }^{19}$ L. J. Giling and J. van de Ven, in Advanced Crystal Growth, edited by P. M. Dryburgh, B. Cockayne, and K. G. Barraclough (Prentice Hall, London, 1987), p. 312.

${ }^{20}$ M. H. J. M. de Croon and L. J. Giling, J. Electroehem. Soc. 137(9), 2867 (1990).

${ }^{21}$ R. B. Bird, W. E. Stewart, and E. N. Lightfoot, Transport Phenomena (John Wiley \& Sons, New York, 1960), pp. 19-26 and 249-260.

${ }^{22}$ E. P. Visser, C. R. Kleijn, C. A. M. Govers, C. J. Hoogendoorn, and L. J. Giling, J. Crystal Growth 94(4), 929 (1989).

${ }^{23}$ B. I. Boltaks, in Diffusion in Semiconductors, edited by H. J. Goldsmit (Infosearch, London, 1963), pp. 107-109.

${ }^{24}$ R. B. Bird, W. E. Stewart, and E. N. Lightfoot, Transport Phenomena, 2nd ed. (John Wiley \& Sons, New York, 2002).

${ }^{25}$ R. J. Hamers and Y. Wang, Chem. Rev. 96, 1261 (1996).

${ }^{26}$ R. Konecny and D. J. Doren, J. Phys. Chem. B 101, 10983 (1997).

${ }^{27}$ S. Komatsu and Y. Moriyoshi, J. Appl. Phys. 64(4), 1878 (1988).

${ }^{28}$ M. Söderlund et al., Catal. Lett. 105(3-4), 191 (2005).

${ }^{29}$ T. P. Fehlner and W. S. Koski, J. Am. Chem. Soc. 86, 2733 (1964).

${ }^{30}$ A. B. Baylis, G. A. Pressley, Jr., and F. E. Stafford, J. Am. Chem. Soc. 88, 2428 (1966)

${ }^{31}$ V. Mohammadi, W. B. de Boer, and L. K. Nanver, Appl. Phys. Lett. 101, 111906 (2012).

${ }^{32}$ W. A. Bryant, Thin Solid Films 60, 19 (1979).

${ }^{33}$ A. M. Beers and J. Bloem, Appl. Phys. Lett. 41, 153 (1982). 
${ }^{34}$ K. Shirai and S. Gonda, J. Appl. Phys. 67(10), 6286 (1990)

${ }^{35}$ H. Schlichting, Boundary-Layer Theory, 6th ed. (Mc-Graw-Hill, New York, 1968), pp. 177-178.

${ }^{36}$ S. Middleman and A. K. Hochberg, Process Engineering Analysis in Semiconductor Device Fabrication (McGraw-Hill, Inc., New York, 1993).
${ }^{37}$ D. B. Holmes and J. R. Vermeulen, Chem. Eng. Sci. 23, 717 (1968).

${ }^{38}$ L. J. Giling, J. Electrochem. Soc. 129, 634 (1982).

${ }^{39}$ K. Reinhardt and R. F. Reidy, Cleaning for Semiconductor Manufacturing (John Wiley \& Sons, 2010).

${ }^{40}$ V. Mohammadi, W. B. de Boer, T. L. M. Scholtes, and L. K. Nanver, ECS Trans. 45(6), 39 (2012). 